\title{
Preoperative Sedentary Time Predicts Postoperative Complications in Gastrointestinal Cancer
}

\author{
Takuya Yanagisawa ${ }^{1,2 *}$, Hideshi Sugiura ${ }^{3}$, Noriatsu Tatematsu ${ }^{3}$, Mioko Horiuchi ${ }^{1}$, \\ Saki Migitaka ${ }^{1}$, Keita Itatsu ${ }^{4}$
}

\begin{abstract}
Background: Gastrointestinal cancer has a high global prevalence. Postoperative complications (PCs) affect the length of hospital stay and long-term outcomes. However, it is unclear whether preoperative sedentary time is associated with PCs, independently of physical activity (PA). We aimed to investigate the association between preoperative sedentary time and PCs independently of PA in patients who underwent surgery for gastrointestinal cancer. Methods: In this prospective study, we included 112 patients who underwent colorectal cancer or gastric cancer surgery. Patient characteristics and surgery-related variables were collected. The Japanese version of the International Physical Activity Questionnaire (the usual 7-day short version) was used to assess preoperative PA and sedentary time. Patients were classified into two groups according to the grade of PCs: Clavien-Dindo (CD) grade $<2$ and $\geq 2$. Multivariate logistic regression analysis was performed to identify the risk factors for $\mathrm{CD}$ grade $\geq 2$ PCs. Receiver operating characteristic curve analysis was used to determine the optimal cutoff point of sedentary time for predicting PCs with CD grade 22. Results: PCs occurred in 38 patients (33.9\%). Sedentary time (odds ratio [OR] 1.29, 95\% confidence interval $[\mathrm{CI}]: 1.09-1.53 ; \mathrm{p}<0.01)$ and body mass index (OR 1.17, 95\% CI: 1.01-1.36; $\mathrm{p}=0.03$ ) were associated with PCs independently of total PA. The optimal cutoff point of sedentary time for predicting PCs was $6 \mathrm{~h} /$ day (sensitivity 0.662, specificity 0.658). Conclusion: Preoperative sedentary time is a predictor of PCs in patients who undergo gastrointestinal cancer surgery.
\end{abstract}

Keywords: Sedentary lifestyle- physical activity- postoperative complications- gastrointestinal cancer

Asian Pac J Cancer Prev, 21 (11), 3405-3411

\section{Introduction}

Globally, gastrointestinal cancers, such as colorectal and gastric cancers, are the most frequently diagnosed cancers in both sexes (Bray et al., 2018). Although advances in treatment techniques have improved survival rates in recent years, the incidence of postoperative complications (PCs) in cases with colorectal and gastric cancers is approximately 30\% (Endo et al., 2017). Moreover, PCs affect the hospital length of stay (LOS) (Nakanishi et al., 2018; Zhang et al., 2018) and long-term outcomes, such as overall survival and overall recurrence rate (Law et al., 2007; Li et al., 2018). PCs in gastrointestinal cancer can be predicted by preoperative sarcopenia (Yang et al., 2019), frailty (Vermillion et al., 2017), 6-minute walk distance (6MWD) (Hayashi et al., 2017), fatigue (Heldens et al., 2017), and prognostic nutrition index (PNI) (Kanda et al., 2016). Thus, it is important to clinically assess modifiable factors to prevent PCs.
Physical activity (PA) is an important factor that influences sarcopenia (Cruz-Jentoft et al., 2019) and frailty (Xue et al., 2008). However, while preoperative PA was reported to be associated with PCs in patients with colorectal cancer (Onerup et al., 2019), no such association was observed in another study (Heldens et al., 2017). There are very few reports examining the relationship between preoperative PA and PCs, as most studies have not investigated the intensity of PA. Thus, there is still no consensus on the relationship between preoperative PA and PCs in gastrointestinal cancer.

Sedentary time is one of the PA indicators and is defined as any waking behavior characterized by an energy expenditure of $\leq 1.5$ metabolic equivalents (METs) while in a sitting or reclining posture (Barnes et al., 2012). Sedentary time has been shown to account for approximately $50 \%$ of daily living activities among community-dwelling people (Chen et al., 2018). A Previous meta-analysis indicated associations between

${ }^{1}$ Department of Rehabilitation, Kamiiida Daiichi General Hospital, 2-70 Kamiiida-kitamachi, Kita-ku, Nagoya, Aichi 462-0802, Japan. ${ }^{2}$ Program in Physical and Occupational Therapy, Nagoya University Graduate School of Medicine, 1-1-20 Daiko-minami, Higashi-ku, Nagoya, Aichi 461-8673, Japan. ${ }^{3}$ Department of Integrated Health Sciences, Nagoya University Graduate School of Medicine, 1-1-20 Daiko-minami, Higashi-ku, Nagoya, Aichi 461-8673, Japan. ${ }^{4}$ Department of surgery, Kamiiida Daiichi General Hospital, 2-70 Kamiiida-kitamachi, Kita-ku, Nagoya, Aichi 462-0802, Japan. *For Correspondence: yanagisawa1204@gmail.com 
sedentary time and all-cause mortality, cancer mortality, and cancer incidence, independently of PA (Biswas et al., 2015). Another meta-analysis reported that the mortality risk associated with increased sedentary time could only be eliminated by high levels of moderateintensity PA (Ekelund et al., 2016). These reports suggest the importance of reducing sedentary time in order to prevent adverse outcomes. Moreover, reducing sedentary time rather than encouraging high level PA may be an easier task for patients to handle. Sedentary time is also reportedly associated with sarcopenia (Gianoudis et al., 2015) and frailty (Blodgett et al., 2015), which are risk factors for PCs independently of PA. Preoperative sedentary time is reportedly longer in esophageal cancer patients with than without pulmonary PCs (Feeney et al., 2011). However, the possibility of an association between preoperative sedentary time and PCs independently of PA in gastrointestinal cancer remains unclear.

In the present study, we aimed to investigate the association between preoperative sedentary time and PCs independently of PA in patients with gastrointestinal cancer.

\section{Materials and Methods}

\section{Patients}

In this prospective study, we enrolled 112 patients who underwent open or laparoscopic surgery for primary colorectal $(n=81)$ or gastric cancer $(n=31)$ between October 2016 and December 2019 at Kamiiida Daiichi General Hospital. Exclusion criteria were patients who (1) could not walk unassisted, (2) had cognitive dysfunction, (3) had simultaneous cancer, (4) underwent palliative surgery, and (5) had missing data. This study was approved by the Ethics Committee of Kamiiida Daiichi General Hospital and the Human Research Ethics Committee of the Nagoya University School of Health Sciences. Prior to participation in this study, all patients were provided with a thorough explanation about the study and provided written consent in accordance with the Declaration of Helsinki.

\section{Outcome}

The study outcome was the occurrence of PCs within 30 days after surgery. Clavien-Dindo (CD) classification was used to grade PCs (grade 1-5) (Dindo et al., 2004). To eliminate the possibility of description bias in patient records, grade 1 complications were excluded, and complications above CD grade 2 were considered as PCs.

\section{Preoperative physical activity and sedentary time}

Preoperative PA and sedentary time were assessed using the Japanese version of the usual 7-day short version of the International Physical Activity Questionnaire (IPAQ-SV). This questionnaire is used to assess vigorousto moderate-intensity PA and walking activity during 7 usual days, and sedentary time during the usual a weekday (Craig et al., 2003). Each activity type and intensity score are assigned a METs value according to a published protocol (Sjostrom et al., 2005).

\section{Preoperative physical function, and fatigue}

To assess the physical function, we measured grip strength, usual gait speed, and 6MWD. Grip strength was measured once on each side using a dynamometer (Grip-D, TKK 5401; Takei Scientific Instruments Co., Niigata, Japan), and the average of each pair of measurements was calculated. Usual gait speed was measured over a 10-m distance between the 3- and 13-m marks of a 16-m walkway. To measure the $6 \mathrm{MWD}$, we instructed patients to walk a predetermined course as far as possible for 6 minutes. The distance covered (in meters) by the patients in 6 minutes was described as the 6MWD.

Fatigue was assessed using the Numerical Rating Scale ( 0 , absence of fatigue -10 , maximum fatigue) .

\section{Patient characteristics, surgery-related variables, and data collection}

Age, gender, body mass index (BMI), Brinkman index, presence of polypharmacy, pulmonary function, presence of diabetes mellitus (DM), hypertension, cerebrovascular disease, chronic obstructive pulmonary disease, and heart disease, Eastern Cooperative Oncology Group performance status, cancer type (colorectal or gastric), and pathological TNM stage ( $\mathrm{p}$-stage) were recorded as patient characteristics. Polypharmacy was defined as more than or equal to five daily medications and identified as the predictive factor of PCs (Volakis et al., 2018).

Surgery-related variables including surgical approach (open or laparoscopic), combined resection, operative time, blood loss, and postoperative hospital LOS were recorded.

The preoperative serum levels of albumin, C-reactive protein (CRP), and hemoglobin, white blood cell count, and total lymphocyte count were collected from electronic medical records. The PNI, a nutritional status indicator, was assessed using the equation: PNI $=10 \times$ serum albumin $(\mathrm{mg} / \mathrm{dL})+0.005 \times$ total lymphocyte count (Kanda et al., 2016).

\section{Statistical analysis}

All continuous variables were expressed as median (interquartile ranges). Patients were divided into the following two groups according to the grade of PCs: CD grade $<2$ and $\geq 2$. Intergroup differences were analyzed using the $\chi^{2}$ test or Fisher's exact test for categorical variables and the Mann-Whitney $U$ test for continuous variables. Multivariate logistic regression analysis was performed to identify the risk factors for developing PCs of CD grade $\geq 2$. We used the variables with $p<0.05$ as independent variables in this analysis. Multivariate logistic regression analysis was performed with (model 2) and without (model 1) total PA adjustment. Finally, receiver operating characteristic (ROC) curve analysis was performed to determine the optimal cutoff point of sedentary time. All statistical analyses were performed using EZR version 1.40 (Saitama Medical Center, Jichi Medical University, Saitama, Japan) (Kanda, 2013).

\section{Results}

PCs classified as $\mathrm{CD} \geq 2$ occurred in 38 patients 
(33.9\%). The most frequently observed PC was ileus $(28.9 \%)$, followed by anastomotic leakage and drain retrograde infection (18.4\% each) (Supplementary Table 1).

Patient characteristics and comparisons of measured variables between the $\mathrm{CD}<2$ and $\mathrm{CD} \geq 2$ groups are shown in Table 1. No significant differences were observed between the two groups, except for BMI, sedentary time, operative time, and postoperative hospital LOS. BMI was significantly higher in patients with $C D \geq 2$ than in those with $\mathrm{CD}<2$ group $(\mathrm{p}<0.01)$. Preoperative sedentary time, operative time, and postoperative hospital LOS were significantly longer in patients with $C D \geq 2$ than in those with $\mathrm{CD}<2(\mathrm{p}<0.01, \mathrm{p}=0.01$, and $\mathrm{p}<0.01$, respectively). Combined resection sites were mostly in the gall bladder (Supplemental Table 2).

The results of multivariate logistic regression analysis are shown in Table 2. Sedentary time, BMI, and operative time, all had a value of $\mathrm{p}<0.05$ on univariate analysis, and therefore, underwent multivariate analysis as potential risk factors for PCs. Sedentary time and BMI were identified as risk factors for developing PCs with $\mathrm{CD} \geq 2$ independently

Table 1. Univariate Analysis of Patient Characteristics and Measured Variables in Patients Having PCs with CD Grade $<2$ and $\geq 2$

\begin{tabular}{|c|c|c|c|}
\hline & $\mathrm{CD}<2(\mathrm{n}=74)$ & $\mathrm{CD} \geq 2(\mathrm{n}=38)$ & $\mathrm{p}$ - value \\
\hline Age, years & $71(64-77)$ & $71(63-80)$ & 0.62 \\
\hline Gender, n (male/female) & $44 / 30$ & $27 / 11$ & 0.27 \\
\hline BMI, $\mathrm{kg} / \mathrm{m}^{2}$ & $22.0(19.5-24.6)$ & $24.3(21.6-25.4)$ & $<0.01$ \\
\hline Brinkman index & $345(0-620)$ & $425(0-762)$ & 0.6 \\
\hline Polypharmacy, n (yes/no) & $30 / 44$ & $22 / 16$ & 0.08 \\
\hline$\% \mathrm{VC}, \%$ & $96.3(87.3-108.7)$ & $93.9(85.0-106.7)$ & 0.37 \\
\hline FEV $1.0 \%, \%$ & $78.3(73.5-82.8)$ & $78.5(72.9-83.5)$ & 0.99 \\
\hline PS, n $(0 / 1)$ & $55 / 19$ & $32 / 6$ & 0.23 \\
\hline DM, n (yes/no) & $17 / 57$ & $15 / 23$ & 0.06 \\
\hline HT, n (yes/no) & $37 / 37$ & $20 / 18$ & 0.79 \\
\hline CVD, n (yes/no) & $66 / 8$ & $35 / 3$ & 0.74 \\
\hline COPD, n (yes/no) & $72 / 2$ & $37 / 1$ & $0.99<$ \\
\hline HD, n (yes/no) & $70 / 4$ & $35 / 3$ & 0.68 \\
\hline Cancer type, $\mathrm{n}$ (colorectal/gastric) & $56 / 18$ & $25 / 13$ & 0.26 \\
\hline P-stage, $\mathrm{n}\left(0^{\mathrm{a}}\right)$ & $50 / 24$ & $24 / 14$ & 0.64 \\
\hline PNI & $48.1(43.8-53.3)$ & $49.2(45.1-53.1)$ & 0.6 \\
\hline Albumin, $\mathrm{g} / \mathrm{dL}$ & $4.0(3.7-4.3)$ & $4.0(3.6-4.3)$ & 0.87 \\
\hline $\mathrm{CRP}, \mathrm{mg} / \mathrm{dL}$ & $0.17(0.07-0.48)$ & $0.32(0.13-0.57)$ & 0.07 \\
\hline Hemoglobin, $\mathrm{g} / \mathrm{dL}$ & $12.6(11.3-14.4)$ & $13.1(11.2-14.8)$ & 0.53 \\
\hline $\mathrm{WBC}, \times 10^{3} / \mu \mathrm{L}$ & $6.3(5.3-7.9)$ & $6.5(5.3-7.3)$ & 0.8 \\
\hline $\mathrm{TLC}, \times 10^{3} / \mu \mathrm{L}$ & $1.6(1.2-2.0)$ & $1.7(1.4-2.1)$ & 0.12 \\
\hline Total PA, METs-h/week & $1270(282-2115)$ & $1386(693-2970)$ & 0.26 \\
\hline Vigorous-intensity activity, METs-h/week & $0(0-0)$ & $0(0-0)$ & 0.49 \\
\hline Moderate-intensity activity, METs-h/week & $0(0-240)$ & $0(0-15)$ & 0.53 \\
\hline Walking activity, METs-h/week & $1270(282-2115)$ & $1386(693-2970)$ & 0.41 \\
\hline Sedentary time, $\mathrm{h} /$ day & $4(2.75-7)$ & $6(4-8)$ & $<0.01$ \\
\hline Grip strength, $\mathrm{kg}$ & $25.4(19.9-32.6)$ & $30.9(21.5-35.5)$ & 0.12 \\
\hline Usual gait speed, $\mathrm{m} / \mathrm{s}$ & $1.22(0.97-1.43)$ & $1.32(1.13-1.49)$ & 0.1 \\
\hline $6 \mathrm{MWD}, \mathrm{m}$ & $467(368-531)$ & $460(427-540)$ & 0.36 \\
\hline Fatigue, points & $0(0-3)$ & $0(0-1)$ & 0.47 \\
\hline Surgical approach, n (open/laparoscopy) & $40 / 34$ & $17 / 21$ & 0.35 \\
\hline Combined resection, $\mathrm{n}$ (yes/no) & $12 / 62$ & $9 / 29$ & 0.33 \\
\hline Operative time, $\min$ & $245(189-313)$ & $287(229-407)$ & 0.01 \\
\hline Blood loss, $\mathrm{mL}$ & $89(28-352)$ & $142(39-322)$ & 0.16 \\
\hline Postoperative hospital LOS, days & $11.5(10-14)$ & $21.5(16-32)$ & $<0.01$ \\
\hline
\end{tabular}

a, colorectal cancer only; CD, Clavien-Dindo; PCs, postoperative complications; BMI, body mass index; $\% \mathrm{VC}, \%$ vital capacity; FEV1.0\%, forced expiratory volume in 1 second \%; PS, performance status; DM, diabetes mellitus; HT, hypertension; CVD, cerebrovascular disease; COPD, chronic obstructive pulmonary disease; HD, heart disease; P-stage, pathological stage; PNI, prognostic nutrition index; CRP, C-reactive protein; WBC, white blood cell count; TLC, total lymphocyte count; PA, physical activity; METs, metabolic equivalents; 6MWD, 6-minute walk distance; LOS, length of stay 
Table 2. Multivariate Logistic Regression Analysis for the Risk of PCs with CD Grade $\geq 2$

\begin{tabular}{lcccc}
\hline & \multicolumn{2}{c}{ Model 1 } & Model 2 \\
& OR $(95 \%$ CI) & P-value & OR (95\% CI) & P-value \\
\hline Sedentary time, per 1 h/day & $1.21(1.05-1.40)$ & $<0.01$ & $1.29(1.09-1.53)$ & $<0.01$ \\
BMI, per 1 kg/m ${ }^{2}$ & $1.15(1.00-1.34)$ & 0.05 & $1.17(1.01-1.36)$ & 0.03 \\
Operative time, per 1 min & $1.00(0.99-1.00)$ & 0.09 & $1.00(0.99-1.00)$ & 0.2 \\
\hline
\end{tabular}

Three variables with $\mathrm{p}<0.05$ (i.e., sedentary time, BMI, and operative time) in univariate analysis were entered into the multivariate logistic regression models; Model 2 was adjusted for total PA; CD, Clavien-Dindo; PCs, postoperative complications; OR, odds ratio; CI, confidence interval; BMI, body mass index; PA, physical activity

Table 3. Univariate Analysis of Patient Characteristics and Measured Variables in Patients with Sedentary Time $<6 \mathrm{~h} /$ day and $\geq 6 \mathrm{~h} /$ day

\begin{tabular}{|c|c|c|c|}
\hline & $\begin{array}{l}\text { Sedentary time } \\
\quad<6 \text { h/day } \\
(\mathrm{n}=62)\end{array}$ & $\begin{array}{l}\text { Sedentary time } \\
\quad \geq 6 \text { h/day } \\
(\mathrm{n}=50)\end{array}$ & $\mathrm{p}$ value \\
\hline Age, years & $68.5(63-76.25)$ & $74(66.75-79.25)$ & 0.05 \\
\hline Gender, n (male/female) & $37 / 25$ & $34 / 16$ & 0.36 \\
\hline BMI, $\mathrm{kg} / \mathrm{m}^{2}$ & $23.1(20.0-25.3)$ & $22.7(19.9-24.9)$ & 0.71 \\
\hline Brinkman index & $200(0-570)$ & $500(0-800)$ & 0.02 \\
\hline Polypharmacy, n (yes/no) & $23 / 39$ & $29 / 21$ & 0.02 \\
\hline$\% \mathrm{VC}, \%$ & $96.3(86.7-109.3)$ & $94.8(86.7-106.9)$ & 0.81 \\
\hline FEV $1.0 \%, \%$ & $78.3(73.8-83.6)$ & $78.6(71.6-82.6)$ & 0.81 \\
\hline PS, n (0/1) & $48 / 14$ & $39 / 11$ & 0.94 \\
\hline DM, n (yes/no) & Nov-51 & $21 / 29$ & $<0.01$ \\
\hline HT, n (yes/no) & $28 / 34$ & $29 / 21$ & 0.17 \\
\hline CVD, n (yes/no) & $56 / 6$ & $45 / 5$ & $0.99<$ \\
\hline COPD, n (yes/no) & $61 / 1$ & $48 / 2$ & 0.58 \\
\hline HD, n (yes/no) & $59 / 3$ & $46 / 4$ & 0.69 \\
\hline Cancer type, n (colorectal/gastric) & $49 / 13$ & $32 / 18$ & 0.07 \\
\hline P-stage, $\mathrm{n}\left(0^{\mathrm{a}}\right)$ & $43 / 19$ & $31 / 19$ & 0.41 \\
\hline PNI & $47.6(43.4-53.0)$ & $49.2(46.0-53.4)$ & 0.16 \\
\hline Albumin, $g / d L$ & $4.0(3.6-4.3)$ & $4.0(3.8-4.3)$ & 0.54 \\
\hline $\mathrm{CRP}, \mathrm{mg} / \mathrm{dL}$ & $0.15(0.07-0.48)$ & $0.31(0.13-0.50)$ & 0.03 \\
\hline Hemoglobin, g/dL & $12.5(11.3-14.5)$ & $13.0(11.1-14.9)$ & 0.68 \\
\hline $\mathrm{WBC}, \times 10^{3} / \mu \mathrm{L}$ & $6.2(5.3-7.3)$ & $6.7(5.3-8.0)$ & 0.41 \\
\hline $\mathrm{TLC}, \times 10^{3} / \mu \mathrm{L}$ & $1.6(1.3-1.9)$ & $1.8(1.3-2.3)$ & 0.24 \\
\hline Total PA, METs-h/week & $1827(855-3386)$ & $763(0-1386)$ & $<0.01$ \\
\hline Vigorous-intensity activity, METs-h/week & $0(0-0)$ & $0(0-0)$ & 0.03 \\
\hline Moderate-intensity activity, METs-h/week & $0(0-480)$ & $0(0-0)$ & 0.01 \\
\hline Walking activity, METs-h/week & $1386(684-2772)$ & $459(0-1386)$ & $<0.01$ \\
\hline Grip strength, kg & $27.8(19.0-34.4)$ & $27.3(20.7-33.4)$ & 0.97 \\
\hline Usual gait speed, $\mathrm{m} / \mathrm{s}$ & $1.28(1.04-1.45)$ & $1.18(1.00-1.39)$ & 0.15 \\
\hline 6MWD, m & $475(380-546)$ & $452(373-502)$ & 0.32 \\
\hline Fatigue, points & $0(0-1.5)$ & $0(0-2)$ & 0.71 \\
\hline Surgical approach, n (open/laparoscopy) & $28 / 34$ & $29 / 21$ & 0.17 \\
\hline Combined resection, $\mathrm{n}$ (yes/no) & $11 / 51$ & $10 / 40$ & 0.76 \\
\hline Operative time, $\min$ & $271(195-365)$ & $253(206-339)$ & 0.72 \\
\hline Blood loss, mL & $100(36-319)$ & $115(27-350)$ & 0.78 \\
\hline PCs, n (yes/no) & $13 / 49$ & $25 / 25$ & $<0.01$ \\
\hline Postoperative hospital LOS, days & $12(10-18)$ & $14.5(10-22)$ & 0.4 \\
\hline
\end{tabular}

a, colorectal cancer only; BMI, body mass index; $\% \mathrm{VC}, \%$ vital capacity; FEV1.0\%, forced expiratory volume in 1 second $\%$; PS, performance status; DM, diabetes mellitus; HT, hypertension; CVD, cerebrovascular disease; COPD, chronic obstructive pulmonary disease; HD, heart disease; P-stage, pathological stage; PNI, prognostic nutrition index; CRP, C-reactive protein; WBC, white blood cell count; TLC, total lymphocyte count; PA, physical activity; METs, metabolic equivalents; 6MWD, 6-minute walk distance; PCs, postoperative complications; LOS, length of stay. 


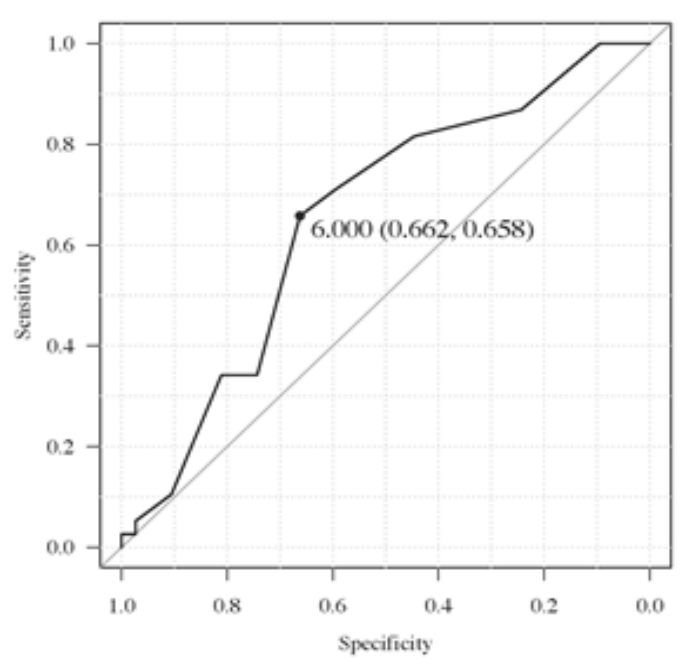

Figure 1. The Receiver Operating Characteristic Curve of Preoperative Sedentary Time for Predicting PCs with $\mathrm{CD}$ Grade $\geq 2$. The area under the curve was $0.65(95 \%$ CI: $0.55-0.75$ ).

of total PA. The respective odds ratios for the occurrence of PCs with $\mathrm{CD} \geq 2$ were 1.29 (95\% confidence interval $[\mathrm{CI}]: 1.09-1.53 ; \mathrm{p}<0.01)$ and 1.17 (95\%CI: 1.01-1.36; $\mathrm{p}=0.03)$ in increments of $1 \mathrm{~h} /$ day for sedentary time and $1 \mathrm{~kg} / \mathrm{m}^{2}$ for BMI (Table 2).

The area under the ROC curve for preoperative sedentary time was 0.65 (95\% CI: $0.55-0.75 ; \mathrm{p}<0.01)$. The optimal cutoff point of preoperative sedentary time for predicting PCs with CD grade $\geq 2$ was $6 \mathrm{~h} /$ day (sensitivity 0.662 , specificity 0.658 ) (Figure 1).

Patient characteristics and comparisons of measured variables between patients with sedentary time $<6 \mathrm{~h} /$ day and those with sedentary time $\geq 6 \mathrm{~h} /$ day are shown in Table 3. Brinkman index and CRP were significantly higher in patients with sedentary time $\geq 6 \mathrm{~h}$ /day compared to those with sedentary time $<6 \mathrm{~h} /$ day $(\mathrm{p}=0.02$ and $\mathrm{p}=0.03$, respectively). Significantly higher numbers of patients with sedentary time $\geq 6 \mathrm{~h} /$ day were exposed polypharmacy, had DM, and developed PCs with CD grade $\geq 2$ compared to those with sedentary time $<6 \mathrm{~h} /$ day $(\mathrm{p}=0.02, \mathrm{p}<0.01$, and $\mathrm{p}<0.01$, respectively). Total $\mathrm{PA}$, vigorous-intensity activity, moderate-intensity activity, and walking activity were significantly fewer in patients with sedentary time $\geq 6 \mathrm{~h} /$ day than those with sedentary time $<6 \mathrm{~h} /$ day $(\mathrm{p}<0.01$, $\mathrm{p}=0.03, \mathrm{p}=0.01$, and $\mathrm{p}<0.01$, respectively).

\section{Discussion}

The present study demonstrated that PCs with CD grade $\geq 2$ in patients who underwent gastrointestinal cancer surgery could be predicted by preoperative sedentary time independently of total PA. Thus, preoperative sedentary time might be a useful screening tool to identify patients at a high risk of PCs with $\mathrm{CD}$ grade $\geq 2$ following gastrointestinal cancer surgery. To our knowledge, the present study is the first to reveal the relationship between preoperative sedentary time and PCs, independently of total PA, in patients who underwent gastrointestinal cancer surgery.

In the present study, Brinkman index, CRP, and the number of patients exposed to polypharmacy and had DM were significantly higher among patients with sedentary time $\geq 6 \mathrm{~h} /$ day compared to those with sedentary time $<6 \mathrm{~h} /$ day. In previous studies, sedentary time, as assessed by a questionnaire, was found to be associated with insulin resistance, inflammatory markers, and the number of medications (Celis-Morales et al., 2012; Yates et al., 2012; Heseltine et al., 2015). Moreover, the preoperative Brinkman index, polypharmacy, DM, and CRP were associated with PCs (Frisch et al., 2010; Kubo et al., 2013; Xue et al., 2018; Yoshikawa et al., 2019). Previous experimental studies have indicated that even short-term sedentary behavior affects systemic function. Restaino et al., (2015) reported that sitting for 6 hours markedly reduced lower leg micro- and macrovascular dilator function in young healthy men. Hamburg et al., (2007) reported that 5-day bed rest increased insulin resistance, serum glucose, total cholesterol, and systolic blood pressure, and decreased calf blood flow in healthy volunteers. It was speculated that prolonged sedentary time affected the systemic functions and increased the risk of PCs; however, the detailed mechanisms are unknown and require further study.

In the present study, the optimal cutoff point of preoperative sedentary time for predicting PCs with CD grade $\geq 2$ was $6 \mathrm{~h} /$ day, with a sensitivity of $66.2 \%$ and specificity of $65.8 \%$. One previous study reported that individuals with sedentary time $\geq 6 \mathrm{~h} /$ day had a higher risk of metabolic syndrome than those with sedentary time $<6 \mathrm{~h}$ /day (Petersen et al., 2014). Another study showed that individuals with sedentary time $>5 \mathrm{~h} /$ day had higher all-cause mortality than those with sedentary time $\leq 5 \mathrm{~h}$ /day (Larsson and Wolk, 2019). Thus, we believe that our cutoff value $(6 \mathrm{~h} /$ day) is clinically significant in predicting PCs notwithstanding its low sensitivity and specificity. According to Coqueiro (2017), the cutoff point of sedentary time as assessed by IPAQ-SV was $7 \mathrm{~h}$ /day for predicting frailty; according to Ohashi (2018), that for predicting sarcopenia was $8 \mathrm{~h} /$ day. The optimal cutoff value may vary depending on the patient characteristics and outcomes; therefore, further research is warranted.

We also revealed an association between BMI and PCs with $\mathrm{CD}$ grade $\geq 2$. A previous meta-analysis reported that BMI was associated with PCs in patients with colorectal (Almasaudi et al., 2018) and gastric cancer (Zhao et al., 2018); which corroborates with our study results. In previous studies, operative time and blood loss were significantly higher in high than in low BMI patients (Hede et al., 2015; Feng et al., 2018), suggesting that a high BMI might predispose patients to more surgical stress.

In the present study, sedentary time was associated with PCs, unlike preoperative PA, grip strength, usual gait speed, 6MWD, PNI, and fatigue. This finding differed from those of previous studies (Kanda et al., 2016; Hayashi et al., 2017; Heldens et al., 2017; Onerup et al., 2019). Possibly, that physical functioning and nutritional status may not directly reflect more sedentary time and a lower level of PA. The results of the present study may suggest the importance of reducing sedentary time rather than exercise intervention to prevent PCs in patients without sarcopenia or frailty, who have no or minimal 
physical function or nutritional status decline.

Our study has several limitations. First, the results may not adequately adjust for confounding factors due to a small number of patient sample size. Thus, our results must be interpreted with caution. Second, the present study included patients who underwent colorectal surgery and gastric surgery. Further studies are necessary to separately analyze patients with colorectal and gastric cancer. Finally, it was not possible to study the different types of PCs because of the small patient sample size. It is necessary to conduct further large-scale studies to analyze PCs by type.

In conclusion, preoperative sedentary time could predict PCs in patients who underwent surgery for gastrointestinal cancer.

\section{Acknowledgements}

We thank all patients who participated in the present study. We also thank collaborating surgeons and other medical staff for their important contributions to our study and Editage (www.editage.com) for English language editing.

\section{Funding Statement}

This work was supported in part by JSPS KAKENHI (Grant Number 18H03127 to H. Sugiura) from Japan Society for the Promotion Science.

\section{References}

Almasaudi AS, McSorley ST, Edwards CA, McMillan DC (2018). The relationship between body mass index and short term postoperative outcomes in patients undergoing potentially curative surgery for colorectal cancer: A systematic review and meta-analysis. Crit Rev Oncol Hematol, 121, 68-73.

Barnes J, Behrens TK, Benden ME, et al (2012). Letter to the editor: standardized use of the terms "sedentary" and "sedentary behaviours". Appl Physiol Nutr Metab, 37, 540-2.

Biswas A, Oh PI, Faulkner GE, et al (2015). Sedentary time and its association with risk for disease incidence, mortality, and hospitalization in adults: a systematic review and meta-analysis. Ann Intern Med, 162, 123-32.

Blodgett J, Theou O, Kirkland S, Andreou P, Rockwood K (2015). The Association between sedentary behaviour, moderate-vigorous physical activity and frailty in NHANES cohorts. Maturitas, 80, 187-91.

Bray F, Ferlay J, Soerjomataram I, et al (2018). Global cancer statistics 2018: GLOBOCAN estimates of incidence and mortality worldwide for 36 cancers in 185 countries. $C A$ Cancer J Clin, 68, 394-424.

Celis-Morales CA, Perez-Bravo F, Ibañez L, et al (2012). Objective vs. self-reported physical activity and sedentary time: effects of measurement method on relationships with risk biomarkers. PLoS One, 7, e36345.

Chen T, Kishimoto H, Honda T, et al (2018). Patterns and levels of sedentary behavior and physical activity in a general Japanese population: The Hisayama Study. J Epidemiol, 28, 260-5.

Coqueiro RD, de Queiroz BM, Oliveira DS, et al (2017). Cross-sectional relationships between sedentary behavior and frailty in older adults. J Sports Med Phys Fitness, 57, 825-30.

Craig CL, Marshall AL, Sjöström M, et al (2003). International physical activity questionnaire: 12-country reliability and validity. Med Sci Sports Exerc, 35, 1381-95.

Cruz-Jentoft AJ, Bahat G, Bauer J, et al (2019). Sarcopenia: revised European consensus on definition and diagnosis. Age Ageing, 48, 16-31.

Dindo D, Demartines N, Clavien PA (2004). Classification of surgical complications: a new proposal with evaluation in a cohort of 6336 patients and results of a survey. Ann Surg, 240, 205-13.

Ekelund U, Steene-Johannessen J, Brown WJ (2016). Does physical activity attenuate, or even eliminate, the detrimental association of sitting time with mortality? A harmonized meta-analysis of data from more than 1 million men and women. Lancet, 388, 1302-10.

Endo T, Momoki C, Yamaoka M, et al (2017). Validation of skeletal muscle volume as a nutritional assessment in patients with gastric or colorectal cancer before radical surgery. $J$ Clin Med Res, 9, 844-59.

Feeney C, Reynolds JV, Hussey J (2011). Preoperative physical activity levels and postoperative pulmonary complications post-esophagectomy. Dis Esophagus, 24, 489-94.

Feng F, Zheng G, Guo X, et al (2018). Impact of body mass index on surgical outcomes of gastric cancer. BMC Cancer, 18, 151.

Frisch A, Chandra P, Smiley D, et al (2010). Prevalence and clinical outcome of hyperglycemia in the perioperative period in noncardiac surgery. Diabetes Care, 33, 1783-8.

Gianoudis J, Bailey CA, Daly RM (2015). Associations between sedentary behaviour and body composition, muscle function and sarcopenia in community-dwelling older adults. Osteoporos Int, 26, 571-9.

Hamburg NM, McMackin CJ, Huang AL, et al (2007). Physical inactivity rapidly induces insulin resistance and microvascular dysfunction in healthy volunteers. Arterioscler Thromb Vasc Biol, 27, 2650-6.

Hayashi K, Yokoyama Y, Nakajima H, et al (2017). Preoperative 6-minute walk distance accurately predicts postoperative complications after operations for hepato-pancreato-biliary cancer. Surgery, 161, 525-32.

Hede P, Sörensson MÅ, Polleryd P, Persson K, Hallgren T (2015). Influence of BMI on short-term surgical outcome after colorectal cancer surgery: a study based on the Swedish national quality registry. Int $J$ Colorectal Dis, 30, 1201-7.

Heldens AFJM, Bongers BC, Lenssen AF, et al (2017). The association between performance parameters of physical fitness and postoperative outcomes in patients undergoing colorectal surgery: An evaluation of care data. Eur J Surg Oncol, 43, 2084-92.

Heseltine R, Skelton DA, Kendrick D, et al (2015). Keeping Moving": Factors associated with sedentary behaviour among older people recruited to an exercise promotion trial in general practice. BMC Fam Pract, 16, 67.

Kanda M, Mizuno A, Tanaka C, et al (2016). Nutritional predictors for postoperative short-term and long-term outcomes of patients with gastric cancer. Medicine (Baltimore), 95, e3781.

Kanda Y (2013). Investigation of the freely available easy-to-use software 'EZR' for medical statistics. Bone Marrow Transplant, 48, 452-8.

Kubo T, Ono S, Ueno H, et al (2013). Elevated preoperative $\mathrm{C}$-reactive protein levels are a risk factor for the development of postoperative infectious complications following elective colorectal surgery. Langenbecks Arch Surg, 398, 965-71.

Larsson SC, Wolk A (2019). Sedentary leisure-time in relation to mortality and survival time. J Sci Med Sport, 22, 562-7.

Law WL, Choi HK, Lee YM, Ho JW (2007). The impact of postoperative complications on long-term outcomes 
following curative resection for colorectal cancer. Ann Surg Oncol, 14, 2559-66.

Li Z, Bai B, Ji G, Li J, Zhao Q (2018). Relationship between Clavien-Dindo classification and long-term survival outcomes after curative resection for gastric cancer: A propensity score-matched analysis. Int J Surg, 60, 67-73.

Nakanishi R, Oki E, Sasaki S, et al (2018). Sarcopenia is an independent predictor of complications after colorectal cancer surgery. Surg Today, 48, 151-7.

Ohashi K, Ishikawa T, Hoshi A, et al (2018). Relationship between sarcopenia and both physical activity and lifestyle in patients with chronic liver disease. J Clin Med Res, 10, 920-7.

Onerup A, Angenete E, Bonfre P, et al (2019). Self-assessed preoperative level of habitual physical activity predicted postoperative complications after colorectal cancer surgery: A prospective observational cohort study. Eur J Surg Oncol, 45, 2045-51.

Petersen CB, Nielsen AJ, Bauman A, Tolstrup JS (2014). Joint association of physical activity in leisure and total sitting time with metabolic syndrome amongst 15,235 Danish adults: a cross-sectional study. Prev Med, 69, 5-7.

Restaino RM, Holwerda SW, Credeur DP, Fadel PJ, Padilla J (2015). Impact of prolonged sitting on lower and upper limb micro- and macrovascular dilator function. Exp Physiol, 100, 829-38.

Sjostrom M, Ainsworth B, Bauman A, et al (2005). Guidelines for data processing analysis of the International Physical Activity Questionnaire (IPAQ) - Short and long forms. https://docs.google.com/viewer? $\mathrm{a}=\mathrm{v} \&$ pid $=$ sites\&srcid=ZG VmYXVsdGRvbWFpb-nx0aGVpcGFxfGd4OjE0NDgxMD k3NDU1YWRIZTM. Accessed 15 January 2020.

Vermillion SA, Hsu FC, Dorrell RD, Shen P, Clark CJ (2017). Modified frailty index predicts postoperative outcomes in older gastrointestinal cancer patients. J Surg Oncol, 115, 997-1003.

Volakis KA, Thorand B, Peters A, et al (2018). Physical activity, muscular strength, and polypharmacy among older multimorbid persons: Results From the KORA-Age Study. Scand J Med Sci Sports, 28, 604-12.

Xue DD, Cheng Y, Wu Mei, Zhang Y (2018). Comprehensive geriatric assessment prediction of postoperative complications in gastrointestinal cancer patients: a meta-analysis. Clin Interv Aging, 13, 723-36.

Xue QL, Bandeen-Roche K, Varadhan R, Zhou J, Fried LP (2008). Initial manifestations of frailty criteria and the development of frailty phenotype in the Women's Health and Aging Study II. J Gerontol A Biol Sci Med Sci, 63, 984-90.

Yang J, Zhang T, Feng D, et al (2019). A new diagnostic index for sarcopenia and its association with short-term postoperative complications in patients undergoing surgery for colorectal cancer. Colorectal Dis, 21, 538-47.

Yates T, Khunti K, Wilmot EG, et al (2012). Self-reported sitting time and markers of inflammation, insulin resistance, and adiposity. Am J Prev Med, 42, 1-7.

Yoshikawa R, Katada J (2019). Effects of active smoking on postoperative outcomes in hospitalised patients undergoing elective surgery: a retrospective analysis of an administrative claims database in Japan. BMJ Open, 9, e029913.

Zhang WT, Lin J, Chen WS, et al (2018). Sarcopenic obesity is associated with severe postoperative complications in gastric cancer patients undergoing gastrectomy: a Prospective Study. $J$ Gastrointest Surg, 22, 1861-9.

Zhao B, Zhang J, Mei D, et al (2018). Does high body mass index negatively affect the surgical outcome and long-term survival of gastric cancer patients who underwent gastrectomy: A systematic review and meta-analysis. Eur J Surg Oncol,
44, 1971-81.

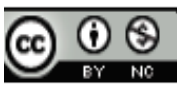

This work is licensed under a Creative Commons AttributionNon Commercial 4.0 International License. 